\title{
Exercício da Atenção e Resolução de Problemas em Atividades Cotidianas da Infância
}

\author{
Daniela Karine Ramos ${ }^{1}$
}

${ }^{1}$ Universidade Federal de Santa Catarina, Florianópolis, SC

\begin{abstract}
Resumo
Este trabalho investiga a relação entre as atividades cotidianas desempenhadas na infância e as funções cognitivas de resolução de problemas e de atenção. Dentre as atividades cotidianas, analisaram-se a leitura, a prática de esportes, o acesso ao computador e a interação com games. Para tanto, aplicou-se um questionário organizado em três partes, contemplando dados do perfil das crianças, o registro da frequência das atividades cotidianas consideradas e a avaliação da presença de comportamentos tomados como indicadores do desempenho da capacidade de resolução de problema e de atenção. O questionário foi respondido pelos responsáveis das crianças, considerando sua percepção. Analisaram-se os resultados de uma amostra de 162 crianças na faixa etária de 6 a 11. Os resultados indicaram a associação entre a leitura e a atenção, e entre o acesso ao computador e o jogar games com a capacidade de resolução de problemas.
\end{abstract}

Palavras-chave: funções cognitivas, games, leitura, prática de esporte

Exercise for Attention and Problem Solving in the Daily Activities of Childhood

\begin{abstract}
This study investigates the relation between the daily activities performed in childhood and the cognitive functions of problem solving and attention. Among daily activities, reading, sports practice, computer access, and interaction with games were analyzed. Therefore, a questionnaire was organized in three parts, including data on the children's profile, the record of the frequency of the daily activities and the evaluation of the presence of behaviors taken as indicators of problem solving and attention performance. The questionnaire was answered by the children's guardians, considering their perception. We analyzed data results from a sample of 162 children aged 6-11. The results revealed the association between reading and attention, and between computer access and playing games with problem-solving ability.

Keywords: cognitive functions; digital games; reading; sports practice
\end{abstract}

Ejercicio de Atención y Resolución de Problemas en las Actividades Cotidianas de la Infância

\begin{abstract}
Resumen
Este trabajo investiga la relación entre las actividades diarias desempeñadas en la infancia y las funciones cognitivas de resolución de problemas y de atención. Entre las actividades diarias, se analizaron: lectura, práctica de deportes, acceso a computador e interacción con juegos. Para verificar si hay asociaciones significativas se aplicó un cuestionario organizado en tres partes, 1) contempla los datos de perfil de los niños,2) el registro de frecuencia de las actividades diarias consideradas y 3) evaluación de la presencia de comportamientos, tomados como indicadores del desempeño de la capacidad de resolución de problemas y de atención. El cuestionario fue respondido por los responsables de los niños, teniendo en cuenta su percepción. Se analizaron las respuestas de una muestra de 162 niños con edades entre 6 a 11 años. Los resultados indicaron la asociación entre lectura y atención y entre acceso al computador y juegos con capacidades de resolución de problemas.

Palabras clave: funciones cognitivas; juegos digitales; lectura; práctica de deportes
\end{abstract}

\section{Introdução}

Este trabalho investiga a relação entre as atividades cotidianas da infância e as funções cognitivas de resolução de problemas e de atenção. Dentre as atividades cotidianas, analisam-se hábitos relacionados à leitura, a prática de esportes, o acesso ao computador e a interação com games, para verificar se há associações significativas com os comportamentos, partindo da avaliação realizada pelos responsáveis das crianças participantes, indicativos do desempenho da capacidade de resolução de problemas e da atenção.
Ao abordar atividades cotidianas, o estudo abrange diversos aspectos e áreas para contextualizar as principais atividades analisadas. Além disso, abordamse as funções executivas que integram uma diversidade de habilidades cognitivas (Diamond, 2013), abordam alguns construtos que também estão relacionados, como, por exemplo, a memória, mas que não são analisados. O estudo desenvolvido analisou alguns aspectos relacionados ao cotidiano, mais especificamente, sobre as habilidades de resolução de problemas e atenção.

Nesse sentido, parte-se da ideia que há aspectos fundamentais, como alimentação e prática de exercício 
físico, que influenciam no funcionamento cerebral e no desenvolvimento cognitivo, consequentemente, na aprendizagem, favorecendo-a ou dificultando-a (Sigman, Peña, Goldin, \& Ribeiro, 2014). Estudos reforçam que alguns hábitos ou atividades contribuem com o aprimoramento das funções cognitivas, como a interação com games (Boot, Kramer, Simons, Fabiani, \& Gratton, 2008; Dye \& Bavelier, 2010; Castellar, All, Marez, \& Van Looy, 2015), o hábito da leitura (Sequeira, 1988; Cain, Compton, \& Parrila, 2017) e a atividade física (Silva, Oliveira, \& Helene, 2013; Merege Filho et al., 2013).

Dentre as funções cognitivas, analisaram-se a atenção e a capacidade de resolução de problemas, por integrarem as funções executivas e estarem fortemente vinculadas à aprendizagem e a tarefas do contexto escolar (Diamond, 2013). A atenção pode ser considerada como processo por meio do qual estímulos relevantes são selecionados, separando-os de outros, para então serem transmitidos e processados (Lane \& Pearson, 1982; Gazzaniga, 2005; Lent, 2005; Diamond, 2013).

$\mathrm{Na}$ perspectiva das funções executivas, a atenção pode ser relacionada a vários processos cognitivos. Dentre eles, destacam-se a capacidade de planejamento, a realização de atividades direcionadas com objetos e a conclusão de atividades (Akshoomoff, 2002). A atenção pode, ainda, ser relacionada à resolução de problemas. Segundo Matlin (2004), essa habilidade está fortemente envolvida na compreensão do problema, ao envolver a necessidade de foco às informações importantes.

A atenção modifica-se ao logo do desenvolvimento até a adolescência, tanto pelo desenvolvimento do cérebro, como pelo impacto das experiências e de atividades específicas (Courage \& Richards, 2008). O desenvolvimento da atenção relaciona-se com a capacidade de ignorar informações irrelevantes, e há diferenças quantitativas e qualitativas na operação de atenção seletiva de acordo com a idade das crianças: quanto mais velhas, melhor é a capacidade das crianças atenderem seletivamente a estímulos específicos (Lane \& Pearson, 1982).

Os resultados de um estudo desenvolvido por Egami et al. (2015), por meio da aplicação de uma bateria de tarefas pelo computador com 144 crianças com desenvolvimento típico com idades entre 7 e 12 anos, organizadas em sete grupos conforme a faixa etária, indicam que há rápidas melhorias na atenção sustentada e visual na faixa etária entre 7 e 10 anos de idade, diferente da mudança que ocorre dos 10 aos 12 anos de idade, a qual se dá de maneira mais gradual. Desse modo, pode-se inferir o quanto hábitos e práticas cotidianas podem ter maior influência sobre o desenvolvimento da atenção entre os 7 e 10 anos. Dentre as funções executivas, o controle inibitório envolve a capacidade de controlar a atenção, o comportamento, os pensamentos e as emoções para fazer o que é mais apropriado ou necessário, contribuindo para a contenção de impulsos e a modificação de hábitos (Diamond, 2013).

Nesse sentido, o estudo de Simonds, Kieras, Rueda e Rothbart (2007) investigou crianças de 7 a 10 anos de idade, considerando um questionário dirigido aos pais que mediu o controle de esforço temperamental (capacidade de inibir uma resposta dominante, para realização de uma resposta sub-dominante, visando detectar erros e se envolver no planejamento), em uma tarefa de conflito, avaliando a eficiência da atenção executiva em resposta a um presente indesejável. Os resultados revelaram que a atenção executiva para um presente indesejado recebido aumenta ao longo da idade, bem como a atenção pôde ser relacionada tanto ao controle de esforço temperamental relatado pelos pais, quanto ao sorriso, sugerindo ligações entre as capacidades de atenção, as medidas de temperamento amplo e as situações sociais que exigem controle da atenção (Simonds et al., 2007).

O aumento da autorregulação e do autocontrole na infância são fatores importantes para o desenvolvimento da atenção (Akshoomoff, 2002). A autorregulação está relacionada ao controle do comportamento social e da atenção executiva, envolvendo o direcionamento de esforço e a capacidade de inibir uma resposta dominante, a fim de realizar uma resposta subdominante, para detectar erros e se engajar no planejamento (Rothbart \& Rueda, 2005).

Estudos também encontraram evidências da associação entre a atenção e a inteligência. Cowan, Fristoe, Elliott, Brunner e Saults (2006), ao realizarem um experimento com 52 crianças (10 a 11 anos) e 52 estudantes universitários. O experimento consistiu na avaliação da atenção, da aptidão verbal e não verbal, por meio da aplicação de vários testes e provas, incluindo procedimentos, como testes auditivos e visuais, tarefas de identificação de sons e letras, testes de vocabulário e de análise de padrões. A análise comparativa entre os dois grupos (crianças e adultos) revelou, dentre outros resultados, que a atenção pode ser um preditor de inteligência nas crianças. Tais evidências apoiam-se na comparação e análises realizadas das medidas indicadoras do controle da atenção, da aptidão verbal e não verbal. 
De outro modo, Richards, Samuels, Turnure e Ysseldyke (1990) com o objetivo de investigar até que ponto os alunos com dificuldades de aprendizagem são capazes de sustentar a atenção e se processam informações diferentemente de outros estudantes. Para tanto, realizaram um estudo correlacional sobre a atenção sustentada e seletiva com 30 alunos de quarta, quinta e sexta série com dificuldades de aprendizagem e 20 alunos sem dificuldades em um grupo controle. A aplicação de um teste que avaliou o desempenho contínuo não revelou diferenças entre os dois grupos, sugerindo capacidade similar para manter a atenção e inibir a resposta impulsiva, porém algumas tarefas de atenção seletiva indicaram que os alunos com dificuldades de aprendizagem cometiam mais erros e tiveram tempos de resposta mais longos, sugerindo que são menos capazes de estreitar o foco de sua atenção.

Os estudos de Cowan et al. (2006) e Richards et al. (1990) apresentam resultados diferentes, o que pode ser justificado pelo perfil dos participantes, já que, no primeiro estudo, não se tem crianças ou adultos com dificuldades de aprendizagem. Assim, intui-se que a atenção pode ser um preditor de inteligência ou desempenho em algumas tarefas específicas e quando se observam sujeitos sem transtornos ou comprometimentos no processo aprendizagem. Ao mesmo tempo há que se considerar que os instrumentos utilizados são distintos e que a atenção é um construto influenciado por muitos fatores, por isso os resultados obtidos podem ser relacionados às características dos participantes, ao contexto da pesquisa e ao tipo de tarefa proposta no experimento para avaliação.

No amplo espectro de atividades e tarefas que envolvem a atenção, destaca-se a prática da leitura, pois se refere a uma capacidade cognitiva que requer a atenção e a memória, funções que se desenvolvem ao longo da vida e podem ser associadas à inteligência, à cultura e à experiência do sujeito (Sequeira, 1988). De acordo com Cain, Compton e Parrila (2017), o objetivo da leitura é extrair significado. A leitura envolve compreensão, reflexão e engajamento. Essa atividade permite alcançar objetivos, desenvolver conhecimentos e participar da sociedade, o que envolve a execução e a integração de muitos processos cognitivos. Nessa perspectiva, um estudo com o objetivo de examinar o impacto de quatro manipulações do texto sobre a eficiência da compreensão de leitura, realizado com dois grupos de adolescentes (um de participantes com TDAH e outro controle sem diagnóstico de TDAH) foram avaliados e comparados em quatro condições diferentes (padrão impresso, espaçado impresso, padrão na tela do computador, espaçados na tela do computador) (Stern \& Shalev, 2013). Os resultados indicaram que há uma correlação significativa entre atenção sustentada e compreensão de leitura.

A atenção sustentada envolve a capacidade de o sujeito manter e dirigir seu foco de atenção a um estímulo dentre vários distratores (Rueda, 2013). Por isso, a atividade de leitura supõe o exercício da atenção sustentada como condição para se atribuir sentido ao que é lido e compreender-se o texto.

Assim, ao se considerar que leitura é uma atividade importante que envolve muitos processos cognitivos e sua prática envolve um esforço cognitivo (Matlin, 2004), pode-se supor que o hábito de leitura se constitui como um exercício de habilidades cognitivas, como a atenção. Um estudo desenvolvido por Zhao, Liu, Liu, \& Huang (2018) com 14 crianças leitoras fluentes e outras 14 com dificuldade na fluência da leitura relacionadas à atenção visual, evidenciou que as crianças com dificuldade exibiam menos precisão nas tarefas visuais e diferenças nos tipos de atenção. Nos resultados descritos, observaram-se a correlação entre a atenção visual e a fluência de leitura, indicando que diferentes padrões de atenção podem estar associados a variações nas estratégias de leitura e sugerindo a presença de uma relação próxima entre o processamento cognitivo geral da atenção visuoespacial e do processamento de linguagem.

Destacam-se, ainda, atividades cotidianas que envolvem a prática esportiva por exercitarem diferentes processos cognitivos. Silva, Oliveira e Helene (2013) relatam que funções cognitivas como memória e atenção são importantes para aumentar a eficiência de respostas por meio da antecipação de contingências ambientais conhecidas. De modo geral, estudos indicam que há uma influência positiva da atividade física sobre as funções executivas, como memória, atenção e tempo de reação (Best, 2010; Chaddock, Hillman, Buck, \& Cohen, 2011; Diamond \& Lee, 2011;) e desempenho cognitivo (Merege Filho et al., 2013).

Dentre outras atividades cotidianas que podem influenciar o desenvolvimento das funções cognitivas, tem-se a interação com jogos digitais ou videogames. Estes jogos criam experiências interativas a partir de um conjunto de informações visuais disponíveis para um ou mais jogadores e podem ser acessados a partir de diferentes interfaces, como computadores, consoles e dispositivos móveis, permitindo acesso e interação de acordo com as regras estabelecidas pelo jogo, o que 
pode ir alterando as informações fornecidas aos jogadores (Kirriemuir \& Mcfalane, 2004).

Esses tipos de jogos compartilham características dos jogos analógicos, ao mesmo tempo em que agrega as dinâmicas, a agilidade e o potencial presente no digital. Assim, mesmo o jogo digital possui, de acordo com Prensky (2012), elementos estruturais como regras, metas ou objetivos, resultados, feedback e aspectos relacionados ao conflito, a competição, o desafio ou a oposição. Nos jogos, as regras têm a função de impor limites, estabelecer os caminhos para se chegar aos resultados, mostrar o que é permitido e o que não é (Prensky, 2012; Caillois, 1990).

Ao mesmo tempo, os objetivos e as metas de um jogo têm, sobretudo, a função de motivar o jogador e pautar a mensuração de seu desempenho, quanto mais perto ou longe está de atingir o objetivo ou a meta (Prensky, 2012). Essas características podem ser relacionadas à motivação e ao exercício de funções cognitivas. Nesse sentido, estudos têm apontando para contribuições do acesso a videogames em relação à melhora do aumento na velocidade de reação (Harrar et al., 2014), da atenção e da concentração (Rueda, Checa, \& Cómbita, 2012; Rivero, Quirino, \& Starling-Alver, 2012), da precisão e da rapidez em tarefas que envolvem habilidades aritméticas (Shin, Sutherland, Norris, \& Soloway, 2012) e do desempenho em tarefas de atenção visual (Boot et al., 2008; Schmidt \& Vandewater, 2008; Dye \& Bavelier, 2010).

A interação com os videogames e a prática de esporte envolvem também a resolução de problemas, utilizada em situações em que se pretende atingir determinado objetivo, porém não se tem a solução (Matlin, 2004). A resolução de problemas é caracterizada por três componentes: o estado inicial (situação anterior à resolução), o estado meta (objetivo relacionado à resolução do problema) e os obstáculos (restrições e dificuldades). A resolução de problemas associada na educação, especialmente na área da matemática, envolve a compreensão do texto ou contexto, a recuperação de memórias, a criação de uma nova solução e a decisão sobre quais operações executar (Zheng, Swanson, \& Marcoulides, 2011).

Um estudo realizado por Zheng, Swanson e Marcoulides (2011) com 310 crianças dos anos iniciais do ensino fundamental, pautou-se na aplicação de uma bateria de testes para avaliar o desempenho da resolução de problemas, da memória de trabalho, da leitura e da realização de cálculos matemáticos. Os resultados revelaram que todos os componentes da memória de trabalho têm um papel importante na previsão da precisão da resolução de problemas, mas habilidades básicas adquiridas em domínios acadêmicos específicos (leitura e matemática) podem compensar alguma influência da memória de trabalho na resolução de problemas matemáticos de crianças.

A resolução de problemas envolve a aquisição de informações e sua aplicação, o que pode, em muitas situações, ser encontrado no acesso às tecnologias digitais de informação e comunicação. Diante disso, Zhang, Yu e Wang (2017), ao realizarem um experimento com 32 alunos do quinto ano, descobriram que eles geralmente eram capazes de reconhecer situações em que precisavam de informações adicionais, porém, às vezes, gastavam muito tempo lendo materiais irrelevantes, o que estava significativamente correlacionado com um desempenho pior de resolução de problemas $(r=0,417$, $p=0,018)$. Desse modo, pode-se analisar os impactos que as interações com essas tecnologias no cotidiano têm sobre o desenvolvimento das funções cognitivas.

Diante disso, refuta-se a noção do sujeito como tábula rasa, não se ignora a influência dos aspectos biológicos e genético, privilegiando-se o intercâmbio entre aspectos biológicos e ambientais. Isso porque a experiência e a interação com o meio influem de diversas maneiras e em diferentes intensidades sobre o sistema nervoso (Lent, 2010). Dentre os diferentes estímulos presentes no ambiente externo, destacam-se neste estudo, aspectos e contribuições às funções cognitivas relacionadas à leitura, a prática de esportes e o acesso aos jogos digitais. Esses aspectos constituíram-se como fatores analisados em relação à capacidade de resolução de problemas e à atenção. Define-se, assim, como objetivo deste estudo avaliar se hábitos relacionados à leitura, à prática de esportes, ao acesso ao computador e à interação com games na infância podem ser associados à capacidade de resolução de problemas e à atenção, tomando como base a observação e avaliação realizada pelos responsáveis das crianças participantes. Parte-se da hipótese de que os hábitos analisados repercutem positivamente sobre o desempenho das funções cognitivas, especialmente, da atenção e da capacidade de resolução de problemas.

\section{Método}

Este trabalho caracteriza-se como uma pesquisa exploratória, desenvolvida em uma escola pública federal vinculada a uma universidade. Essa escola atende a um público bem diversificado, pois o acesso dos alunos 
nessa instituição ocorre por sorteio universal, no qual qualquer pessoa pode inscrever seus filhos, respeitando as idades e a documentação exigida.

\section{Participantes}

A amostra da pesquisa foi composta por 162 crianças na faixa etária de 6 a 11 anos. Os participantes estavam distribuídos em sete turmas dos anos iniciais do Ensino Fundamental.

No que se refere às características dos sujeitos participantes, tem-se $52,2 \%$ do sexo feminino e, com média de idade de 8,3 anos $(D P=0,8)$, sendo que $60,08 \%$ tem idades entre 6 e 8 anos. Na Tabela 1, pode-se observar que, das crianças participantes, quase $95 \%$ tinha computador e que o acesso à internet em casa foi superior a $80 \%$.

\section{Instrumentos}

A coleta de dados foi realizada com base na aplicação de um questionário dividido em três partes. $\mathrm{Na}$ primeira, o questionário abordou aspectos relacionados a características de perfil como a idade, o sexo, as pessoas com quem os alunos vivem, se possuem irmão e o acesso às tecnologias: computador, rede wi-fi, tablet ou smartphone.

$\mathrm{Na}$ segunda parte, propôs-se a avaliação da frequência de algumas atividades cotidianas que podem ser consideradas hábitos. Essas atividades e sua frequência foram consideradas variáveis independentes e incluíram: a prática de esporte, o acesso ao computador, o brincar com outras crianças e o jogar videogames.

A terceira parte envolveu a avaliação de comportamentos indicativos das habilidades cognitivas de resolução de problemas e atenção, considerando a percepção dos responsáveis. Cada habilidade foi descrita em termos de 10 comportamentos que poderiam ser observados. Ao analisar cada comportamento, o responsável assinalava uma das opções de uma escala de frequência utilizada. A elaboração das afirmações pautou-se nas definições e caracterização de cada habilidade a partir da fundamentação teórica (Matlin, 2004; Lent, 2010) que foi adaptada para descrição de indicadores e comportamentos manifestos, conforme se observa na Tabela 2.

A partir das respostas obtidas, foi construída uma base de dados em que os comportamentos expressos de forma positiva, como "Consegue manter-se focado quando brinca, joga ou faz algo que tem interesse", recebia pontuação 4 quando assinalado "Sim", 3 quando "Às vezes", 2 quando "Raramente", 1 quando "Não" e 0 para "Não sei". A pontuação era invertida quando o comportamento era expresso de forma negativa, como "Demonstra raiva e impaciência quando não consegue resolver algo", então era atribuído 4 quando assinalado "Não" e 1 quando "Sim", mantendo o valor 0 para "Não Sei". Após essa atribuição, foram somadas as pontuações obtidas em cada habilidade e consideradas como o escore (variável dependente).

A partir da primeira versão do inventário, foi realizado um pré-teste com seis pais, os quais responderam juntamente com o pesquisador e foram questionados sobre a compreensão dos comportamentos descritos para tentar identificar se a descrição permitia compreender o que se pretendia avaliar. Depois desse procedimento, pequenos ajustes foram feitos no instrumento para sua aplicação na pesquisa.

Tabela 1.

Caracterização Sociodemográfica da Amostra

\begin{tabular}{lccc}
\hline & $\mathrm{N}$ & $\%$ & $\mathrm{IC} 95 \%$ \\
\hline Sexo & & & \\
Feminino & 85 & 52,2 & $44,9-59,5$ \\
Masculino & 77 & 47,8 & $40,5-55,1$ \\
\hline Tem computador em casa ${ }^{*}$ & & & \\
Não & 8 & 5,5 & $2,0-9,1$ \\
Sim & 154 & 94,5 & $90,9-98,0$ \\
\hline Tem acesso a internet em casa* & & & \\
Não & 23 & 14,7 & $9,2-20,2$ \\
Sim & 139 & 85,3 & $79,8-90,8$ \\
\hline
\end{tabular}

Nota. * Variável com dados ignorados

Psico-USF, Bragança Paulista, v. 24, n. 4, p. 751-762, out./ dez. 2019 
Tabela 2.

Descrição dos Indicadores Utilizados no Questionário

\begin{tabular}{|c|c|c|c|}
\hline Habilidade & $\begin{array}{l}\text { Quantidade } \\
\text { questões }\end{array}$ & Definição e característica & Exemplo de comportamentos \\
\hline Atenção & 10 & $\begin{array}{l}\text { Envolve focalizar a consciência, } \\
\text { concentrando os processos mentais } \\
\text { em uma única tarefa principal e } \\
\text { colocando as demais em segundo } \\
\text { plano (Lent, 2010). }\end{array}$ & $\begin{array}{l}\text { Consegue manter-se focado durante } \\
\text { a realização das tarefas escolares e } \\
\text { estudo. } \\
\text { Distrai-se com facilidade, qualquer } \\
\text { barulho ou pessoa tira a atenção da } \\
\text { atividade que está sendo realizada. }\end{array}$ \\
\hline $\begin{array}{c}\text { Resolução de } \\
\text { problema }\end{array}$ & 10 & $\begin{array}{c}\text { Envolve a atenção as informações } \\
\text { importantes, pressupondo a seleção } \\
\text { e a decisão sobre quais informações } \\
\text { são mais importantes e vão } \\
\text { contribuir na resolução do problema } \\
\text { (Matlin, 2004). }\end{array}$ & $\begin{array}{l}\text { Antes de começar a fazer alguma } \\
\text { coisa analisa e pensa sobre qual é o } \\
\text { melhor jeito de fazer. } \\
\text { Usa conhecimentos anteriores para } \\
\text { tentar resolver um problema novo. }\end{array}$ \\
\hline
\end{tabular}

\section{Procedimentos}

A coleta e a análise de dados realizada buscou sistematizar a observação do desempenho cognitivo, obtidas pelo registro da percepção dos responsáveis pelas crianças. Essas observações procuraram oferecer indícios das características dos alunos em relação às suas atividades cotidianas, aspectos cognitivos e comportamentos relacionados às habilidades cognitivas de resolução de problemas e atenção. Para tanto, enviou-se o instrumento impresso aos responsáveis pelas professoras das turmas participantes da pesquisa e devolvido na agenda das crianças. Os responsáveis tiveram o prazo de sete dias para responder e devolver.

Por envolver seres humanos, a pesquisa foi submetida e aprovada pelo Comitê de Ética, por meio do parecer 902.620 (CAAE: 33241614.0.0000.0121). Os Termos de Consentimento Livre e Esclarecido (TCLE) foram assinados pelos professores e responsáveis das crianças participantes. Já o Termo de Assentimento foi explicado e assinado pelas crianças em sala de aula.

\section{Análise dos Dados}

Após digitação dos dados no programa Excel, estes foram convertidos em um banco de dados para análise no pacote estatístico Stata versão 11.0 (StataCorp, Texas, USA). As variáveis categóricas foram descritas usando frequências absolutas e relativas, enquanto, para as variáveis numéricas, foram usados a média e o desvio padrão.
Para testar a associação entre as variáveis de comportamento/hábito das crianças com os diferentes escores, foi utilizado o teste de ANOVA, seja de heterogeneidade ou de tendência, dependendo da natureza das variáveis de exposição. Para as análises ajustadas, foi usada a regressão linear múltipla, sendo os resultados apresentados como coeficientes de regressão $(\beta)$ com seus respectivos intervalos de confiança de $95 \%$. O ajuste das associações foi realizado em todos os casos para os seguintes possíveis fatores de confusão: sexo e idade da criança, se mora com a mãe e com o pai, se tem irmãos, presença de computador em casa e disponibilidade de internet no domicílio. Em todos os casos, o nível de significância estatística utilizado foi menor de $5 \%(p<0,05)$.

\section{Resultados}

Os resultados partem da análise dos escores obtidos em relação à atenção e a capacidade de resolução de problemas e sua associação com atividades cotidianas. Analisaram-se atividades como a leitura, o acesso ao computador, a prática de esporte e o acesso a jogos de videogames.

Em relação ao escore de atenção, a média de pontos foi de 33,2 (DP =6,2) de um máximo de 40. A Tabela 3 apresenta os resultados da associação desse desfecho com os comportamentos/atividades da criança. $\mathrm{Na}$ análise bruta, a única variável associada foi o hábito de ler, que teve uma relação direta com o 
escore de atenção, de tal forma que entre aqueles que leem pouco ou nunca, o escore de atenção foi significativamente menor, com diferença de 8,2 pontos $(p<$ $0,05)$ do que naqueles que leem diariamente. Essa tendência se manteve estável após ajuste para variáveis de confusão. Nenhuma das outras variáveis investigadas (prática de esportes, acesso ao computador ou jogar videogame) esteve associada com esse escore, apesar de ser possível observar um aumento crescente na média do escore conforme ocorre o aumento da frequência do comportamento de acesso ao computador ou de jogar videogame.

$\mathrm{Na}$ Tabela 3 podem-se observar os resultados obtidos na associação entre as variáveis relacionadas aos hábitos e suas frequências e os escores obtidos a partir da avaliação feita pelos responsáveis, por meio da análise de correlação.

Em relação ao escore de resolução de problema, a média de pontos foi de 34,7 $(D P=6,4)$ de um valor máximo de 40. A Tabela 4 apresenta os resultados da associação desse desfecho com os comportamentos/ atividades da criança. $\mathrm{Na}$ análise bruta, as variáveis de acesso ao computador e jogar videogames tiveram uma relação direta com o escore de resolução de problemas. As diferenças obtidas nos escores foram estatisticamente significativas $(p>0,05)$, ao comparar-se o escore de quem acessa pouco o computador tem-se a diferença de menos 4,7 pontos do que aqueles que acessam diariamente. Do mesmo modo quem nunca joga videogame tem 3,8 pontos a menos em relação ao escore de

Tabela 3.

Associação entre a Frequência de Comportamentos/Atividades da Criança e o Escore Total de Atenção

\begin{tabular}{|c|c|c|c|c|}
\hline & \multirow{2}{*}{$\mathrm{N}$} & \multicolumn{2}{|c|}{ Análise Bruta } & \multirow{2}{*}{$\frac{\text { Análise ajustada }^{a}}{\beta(\mathrm{IC} 95 \%)}$} \\
\hline & & Média $(D P)$ & $\beta(\mathrm{IC} 95 \%)$ & \\
\hline Hábito de ler & & & $p=0,001 \#$ & $p<0,003 \#$ \\
\hline Diariamente & 48 & $36,7(5,5)$ & Ref & Ref \\
\hline 3-6 dias & 52 & $33,2(5,6)$ & $-3,6(-5,7 ;-1,4)$ & $-4,0(-6,1 ;-1,9)$ \\
\hline 1-2 dias na semana & 43 & $32,1(5,1)$ & $-4,6(-6,9 ;-2,4)$ & $-4,5(-6,7 ;-2,4)$ \\
\hline Pouco no mês/nunca & 21 & $28,5(5,7)$ & $-8,2(-11,0 ;-5,4)$ & $-7,1(-9,9 ;-4,3)$ \\
\hline Prática esportes & & & $p=0,462^{*}$ & $p=0,844^{*}$ \\
\hline Diariamente & 25 & $32,6(6,9)$ & Ref & Ref \\
\hline 3-6 dias & 49 & $33,2(6,4)$ & $0,7(-2,2 ; 3,6)$ & $-0,5(-3,3 ; 2,4)$ \\
\hline 1-2 dias na semana & 56 & $34,3(5,5)$ & $1,7(-1,1 ; 4,6)$ & $0,1(-2,7 ; 2,9)$ \\
\hline Pouco no mês/nunca & 34 & $32,4(5,4)$ & $-0,5(-3,3 ; 3,0)$ & $-1,0(-4,0 ; 2,0)$ \\
\hline Acessa o computador & & & $p=0,333^{*}$ & $p=0,284^{*}$ \\
\hline Diariamente & 76 & $34,1(6,0)$ & Ref & Ref \\
\hline $3-6$ dias & 45 & $32,5(5,1)$ & $-1,6(-3,8 ; 0,7)$ & $-2,0(-4,2 ; 0,3)$ \\
\hline 1-2 dias na semana & 29 & $33,5(6,3)$ & $-0,5(-3,1 ; 2,0)$ & $-0,7(-3,2 ; 1,7)$ \\
\hline Pouco no mês/nunca & 14 & $31,4(7,7)$ & $-2,6(-6,1 ; 0,8)$ & $-2,5(-6,2 ; 1,2)$ \\
\hline Joga games & & & $p=0,779^{*}$ & $p=0,899^{*}$ \\
\hline Diariamente & 42 & $34,0(5,6)$ & Ref & Ref \\
\hline 3-6 dias & 54 & $33,2(5,9)$ & $-0,8(-3,2 ; 1,7)$ & $-0,3(-2,6 ; 2,1)$ \\
\hline 1-2 dias na semana & 41 & $33,7(6,5)$ & $-0,3(-2,9 ; 2,3)$ & $0,1(-2,4 ; 2,6)$ \\
\hline Pouco no mês/nunca & 27 & $32,0(6,1)$ & $-2,0(-4,9 ; 0,9)$ & $-1,0(-4,0 ; 2,0)$ \\
\hline
\end{tabular}

Notas. a - Ajustada para sexo e idade da criança, se mora com a mãe e com o pai, se tem irmãos, presença de computador em casa, uso de UCA em casa e disponibilidade de internet no domicílio. * Teste de Wald de heterogeneidade. \# Teste de Wald de tendência. 
quem joga diariamente, diferença que é maior ainda entre aqueles que jogam pouco no mês ou nunca jogam jogos com peças, que chega a ser de menos 4,6 pontos $(p>0,05)$. Essa tendência se manteve estável após ajuste para variáveis de confusão.

$\mathrm{Na}$ Tabela 4, podem-se observar os resultados obtidos no escore de resolução de problemas em relação à frequência dos hábitos considerados na análise. Observa-se ainda na tabela que as variáveis de prática de esportes e o hábito de brincar com outras crianças não estiveram associadas ao escore de resolução de problemas.

\section{Discussão}

Os resultados obtidos após a análise estatística mostram algumas relações significativas entre atividades cotidianas e o desempenho das funções cognitivas avaliado com base na percepção dos responsáveis. Destacou-se a relação significativa entre o hábito de leitura e os indicadores avaliados pelos pais em relação à atenção. Esse aspecto pode ser associado ao fato de que essa habilidade de leitura envolve fortemente o exercício de outras capacidades cognitivas (Matlin, 2004), ao mesmo tempo em que se supõe que esse resultado reforça as evidências de estudos que descrevem a associação entre a atenção e a compreensão de leitura (Stern \& Shalev, 2013) e a fluência de leitura (Zhao, Liu, Liu, \& Huang 2018).

No presente estudo não foi possível identificar diferenças entre as frequências em que os alunos praticam atividades esportivas e a atenção, apesar da orientação e da manutenção da atenção serem consideradas relevantes ao longo de um evento esportivo (Silva et al., 2013). Apesar de não se evidenciar neste estudo associações com as atividades esportivas, não se pode desconsiderar que essa prática pode oferecer contribuições ao desenvolvimento cognitivo (Sigman et al., 2014). Cabe observar que as respostas obtidas tiverem pouca variação, o que pode remeter a uma característica mais pontual da amostra analisada e indica a necessidade de avaliar melhor o tempo e a intensidade necessária para

Tabela 4.

Associação entre a Frequência das Atividades da Criança e o Escore de Resolução de Problemas

\begin{tabular}{|c|c|c|c|c|}
\hline & $N_{T}$ & & & Análise ajustada ${ }^{a}$ \\
\hline & $N$ & Média $(D P)$ & $\beta(\mathrm{IC95} \%)$ & $\beta(\mathrm{IC} 95 \%)$ \\
\hline Pratica esportes & & & $p=0,467^{*}$ & $p=0,043 \#$ \\
\hline Diariamente & 25 & $35,1(6,5)$ & Ref & Ref \\
\hline 3-6 dias & 49 & $35,6(6,1)$ & $0,5(-2,6 ; 3,7)$ & $-0,4(-3,8 ; 3,0)$ \\
\hline 1-2 dias na semana & 56 & $34,5(5,8)$ & $-0,6(-3,7 ; 2,5)$ & $-2,6(-6,0 ; 0,8)$ \\
\hline Pouco no mês/nunca & 34 & $33,4(7,7)$ & $-1,7(-5,0 ; 1,7)$ & $-2,8(-6,4 ; 0,8)$ \\
\hline & & & $p=0,029 \#$ & $p=0,013 \#$ \\
\hline Acessa o computado & & & & \\
\hline Diariamente & 76 & $35,6(5,7)$ & Ref & Ref \\
\hline 3-6 dias & 45 & $34,3(6,2)$ & $-1,3(-3,7 ; 1,1)$ & $-2,7(-5,4 ; 0,0)$ \\
\hline 1-2 dias na semana & 29 & $34,7(6,6)$ & $-1,0(-3,7 ; 1,8)$ & $-1,2(-4,0 ; 1,7)$ \\
\hline Pouco no mês/nunca & 14 & $30,9(9,3)$ & $-4,7(-8,3 ;-1,0)$ & $-6,8(-11,2 ;-2,5)$ \\
\hline & & & $p=0,014 \#$ & $p=0,016 \#$ \\
\hline Joga games & & & & \\
\hline Diariamente & 42 & $36,7(3,9)$ & Ref & Ref \\
\hline 3-6 dias & 54 & $34,5(5,4)$ & $-2,1(-4,7 ; 0,5)$ & $-2,6(-5,4 ; 0,2)$ \\
\hline 1-2 dias na semana & 41 & $34,0(8,2)$ & $-2,6(-5,4 ; 0,1)$ & $-3,4(-6,3 ;-0,4)$ \\
\hline Pouco no mês/nunca & 27 & $32,9(7,9)$ & $-3,8(-6,9 ;-0,7)$ & $-3,9(-7,4 ;-0,4)$ \\
\hline
\end{tabular}

Notas. a - Ajustada para sexo e idade da criança, se mora com a mão e com o pai, se tem irmãos, presença de computador em casa, uso de UCA em casa e disponibilidade de internet no domicílio. * Teste de Wald de heterogeneidade. \# Teste de Wald de tendência. 
que a prática esportiva possa resultar em mudanças no desempenho das funções cognitivas.

Nos resultados, observou-se, ainda, que o escore da atenção aumentou conforme a frequência de atividades que envolviam o acesso ao computador e o jogar videogames também aumentava. Apesar disso, não se estabeleceu uma relação estatisticamente significativa. Em especial, no que se refere ao uso dos videogames, esse resultado indica que há um potencial para o treino da atenção por meio de seu uso, mas que necessitaria de alguma mediação ou organização mais sistematizada de acesso, conforme algumas pesquisas realizadas descrevem (Diamond, 2012; Baniqued et al., 2013; Waters et al., 2015).

De qualquer modo, destaca-se que há evidências de que treinamentos relacionados à atenção podem ser feitos por meio de atividades como tocar um instrumento ou jogar (Feng, Spence, \& Pratt, 2007; Homer, Plass, Raffaele, Ober, \& Ali, 2018). Isso porque as redes neurais de áreas envolvidas no controle executivo da atenção, quando exercitado e fortalecido por meio do treinamento, produzem melhorias em outras tarefas executivas, incluindo a inteligência geral (Hardiman, Magsamen, McKhann, \& Eilber, 2009).

Os resultados relacionados ao escore de resolução de problemas puderam ser associados a atividades como o acesso ao computador e o jogar videogames. Aspectos que podem ser associados ao fato de que, quando jogamos, faz-se escolhas, adotam-se estratégias, decide-se, por exemplo, se move a peça e qual peça, e que a ação no jogo, resultado dessa escolha, tem um desfecho, ou seja, há uma consequência que muda o jogo (Salen \& Zimmerman, 2012).

Assim, reforça-se o potencial que os jogos digitais possuem para aprimorar a capacidade de resolução de problemas, o que corrobora com resultados obtidos em pesquisas como a realizada por Oei e Patterson (2013), em que se tiveram evidências de associação entre a interação com games e a melhoria em diferentes aspectos cognitivos, incluindo a capacidade de resolução de problemas. Outro estudo, relatado por Rueda, Checa e Cómbita (2012), indica que crianças que participaram de um treinamento computadorizado de atenção ativaram a rede de atenção executiva mais rápido e de forma mais eficiente do que as crianças não treinadas, aspecto que reforça que a interação com os games pode ter influência sobre o desempenho da atenção e da capacidade de resolução de problemas. Ao considerar-se que a interação com videogames envolve um ambiente digital e interativo, os resultados obtidos reforçam a correlação descrita por Zhang et al. (2017) no uso de tecnologias para busca de informações adicionais e o desempenho na resolução de problemas.

De modo geral, as atividades investigadas a partir da percepção dos responsáveis abrangem diferentes atividades cotidianas, as quais incluem a leitura e a prática de esportes, bem como a interação com as tecnologias digitais. A inclusão dessas atividades na pesquisa parte do reconhecimento da importância da diversidade de atividades e experiências para o desenvolvimento infantil (Lent, 2010; Sigman et al., 2014). Entretanto, chama-se a atenção para o fato de que muitas dessas atividades acabam ficando em um segundo plano, já que as crianças e os adolescentes dedicam muito tempo ao acesso de tecnologias digitais, as quais incluem os jogos digitais (Strasburger, Wilson, \& Jordan, 2013).

As preocupações com o impacto do acesso às tecnologias digitais sobre o desenvolvimento de crianças e adolescente não é recente; Subrahmanyam, Greenfield, Kraut e Gross (2001), por exemplo, realizaram uma revisão de pesquisas sobre o impacto do uso de computadores domésticos e relataram pesquisas efetuadas desde a década de 80. Strasburger, Wilson e Jordan (2013) reforçam a preocupação com o excesso de tempo e a falta de regras dos pais, apontando para a necessidade de uma educação, tanto na escola como em casa, em relação ao uso dessas mídias.

\section{Considerações finais}

Os resultados obtidos neste estudo podem ser tomados como indicadores que têm o objetivo de reforçar a importância das diferentes atividades cotidianas para o desenvolvimento cognitivo das crianças, destacando-se a leitura, a prática de esportes e a interação com jogos digitais. As análises revelaram associações que indicam que essas atividades podem contribuir para o aprimoramento de habilidades cognitivas.

Entretanto, por não possuir uma grande abrangência e fazer uso de um instrumento de observação indireta sem controle sobre as percepções, o que pode ter interferência das experiências prévias e da falta de treino dos observadores que responderam ao instrumento, toma-se os resultados apenas como indicadores e ressalta-se que os achados precisariam ser mais bem investigados com base no uso de outros procedimentos e instrumentos.

Apesar disso, os achados reforçam algumas tendências que vêm sendo reveladas em outros estudos e servem como base para maior reflexão sobre como 
atividades cotidianas. Destaca-se, ainda a leitura e o acesso às tecnologias digitais, como o acesso a computadores e aos videogames, pode ter influência sobre o desempenho cognitivo.

Considerando que atualmente tem-se o advento das tecnologias móveis que facilitam o acesso às tecnologias digitais, incluindo as redes sociais e os aplicativos de comunicação instantânea, isso reforça ainda mais a preocupação em relação ao uso intenso dessas tecnologias. Mas este estudo destaca a melhora em relação às funções cognitivas associada à interação com essas tecnologias.

\section{Referências}

Akshoomoff, N. (2002). Selective attention and active engagement in young children. Developmental Neuropsychology, 22(3), 625-642.

Baniqued, P. L., Lee, H., Voss, M. W., Basak, C., Cosman, J. D., DeSouza, S., ... \& Kramer, A. F. (2013). Selling points: What cognitive abilities are tapped by casual video games? Acta psychologica, 142(1), 7486. doi: 10.1016/j.actpsy.2012.11.009

Best, J. R. (2010). Effects of physical activity on children's executive function: Contributions of experimental research on aerobic exercise. Developmental Review, 30(4), 331-351. doi: 10.1016/j. dr.2010.08.001

Boot, W. R., Kramer, A. F., Simons, D. J., Fabiani, M., \& Gratton, G. (2008). The effects of video game playing on attention, memory, and executive control. Acta psychologica, 129(3), 387-398. doi: 10.1016/j.actpsy.2008.09.005

Caillois, Roger. (1990). Os jogos e os homens. Lisboa: Cotovia.

Cain, K., Compton, D. L., \& Parrila, R. K. (Eds.). (2017). Theories of reading development (vol. 15). John Benjamins Publishing Company.

Castellar, E. N., All, A., De Marez, L., \& Van Looy, J. (2015). Cognitive abilities, digital games and arithmetic performance enhancement: A study comparing the effects of a math game and paper exercises. Computers \& Education, 85, 123-133. doi: 10.1016/j.compedu.2014.12.021

Chaddock, L., Hillman, C. H., Buck, S. M., \& Cohen, N. J. (2011). Aerobic fitness and executive control of relational memory in preadolescent children.
Medicine \& Science in Sports \& Exercise, 43(2), 344349. doi: 10.1249/MSS.0b013e3181e9af48

Courage, M. L., \& Richards, J. E. (2008). Attention. Em M. L. M. Haith \& B. J. Benson. Encyclopedia of Infant and Early Childhood Development. Elsevier, 106-117.

Cowan, N., Fristoe, N. M., Elliott, E. M., Brunner, R. P., \& Saults, J. S. (2006). Scope of attention, control of attention, and intelligence in children and adults. Memory \& cognition, 34(8), 1754-1768.

Diamond, A. (2012). Activities and programs that improve children's executive functions. Current $d i$ rections in psychological science, 21(5), 335-341.

Diamond, A. (2013). Executive functions. Annual review of psychology, 64, 135-168. doi: 10.1146/ annurev-psych-113011-143750

Diamond, A., \& Lee, K. (2011). Interventions shown to aid executive function development in children 4 to 12 years old. Science, 333(6045), 959-964. doi: 10.1126/science. 1204529

Dye, M. W., \& Bavelier, D. (2010). Differential development of visual attention skills in school-age children. Vision research, 50(4), 452-459. doi 10.1016/j.visres.2009.10.010

Egami, C., Yamashita, Y., Tada, Y., Anai, C., Mukasa, A., Yuge, K., ... \& Matsuishi, T. (2015). Developmental trajectories for attention and working memory in healthy Japanese school-aged children. Brain and Development, 37(9), 840-848.

Feng, J., Spence, I., \& Pratt, J. (2007). Playing an action video game reduces gender differences in spatial cognition. Psychol Sci, 18(10), 850-855. doi: 10.1111/j.1467-9280.2007.01990.x

Kirriemuir, J., \& McFarlane, A. (2004). Literature review in games and learning. Futurelab. Recuperado de https://hal.archives-ouvertes.fr/hal-00190453/ document

Gazzaniga, M. S. (2005). Ciência Psicológica: Mente, cérebro e comportamento. 2a ed. Porto Alegre: Artmed.

Hardiman, M., Magsamen, S., McKhann, G., \& Eilber, J. (2009). Neuroeducation: Learning, arts, and the brain. New York/Washington, DC: Dana Foundation.

Harrar, V., Tammam, J., Perez-Bellido, A., Pitt, A., Stein, J., \& Spence, C. (2014). Multisensory integration

Psico-USF, Bragança Paulista, v. 24, n. 4, p. 751-762, out./ dez. 2019 
and attention: Differences in dyslexics. Current Biology, 24, 1-5. doi: 10.1016/j.cub.2014.01.029

Homer, B. D., Plass, J. L., Raffaele, C., Ober, T. M., \& Ali, A. (2018). Improving high school students' executive functions through digital game play. Computers \& Education, 117, 50-58. doi: 10.1016/j. compedu.2017.09.011

Lane, D. M., \& Pearson, D. A. (1982). The development of selective attention. Merrill-Palmer Quarterly (1982-), 317-337.

Lent, R. (2010). Cem bilhões de neurônios: Conceitos fundamentais de neurociências. (2a ed.). São Paulo: Atheneu.

Matlin, M.W. (2004). Psicologia cognitiva. (5a ed). Rio de Janeiro (RJ): LTC.

Merege Filho, C. A. A., Alves, C. R. R., Neves, W. D., Junior, A. H. L., Gualano, B., \& dos Santos Costa, A. (2013). Associação entre o nível de atividade física de lazer e o desempenho cognitivo em crianças saudáveis. Revista Brasileira de Educação Física e Esporte, 27(3), 355-361. doi: 10.1590/ S1807-55092013000300003

Oei, A. C., \& Patterson, M. D. (2013). Enhancing cognition with video games: A multiple game training study. PLoS One, 8(3), e58546. doi: 10.1371/journal.pone. 0058546

Prensky, M. (2012). Aprendizagem baseada em jogos digitais. São Paulo: SENAC.

Richards, G. P., Samuels, S. J., Turnure, J. E., \& Ysseldyke, J. E. (1990). Sustained and selective attention in children with learning disabilities. Journal of learning disabilities, 23(2), 129-136.

Rivero, T. S., Querino, E. H., \& Starling-Alves, I. (2012). Videogame: Seu impacto na atenção, percepção e funções executivas. Revista Neuropsicologia Latinoamericana, 1(1), 38-52.

Rothbart, M. K., \& Rueda, M. R. (2005). The development of effortful control. Em U. Mayr, E. Awh \& S. W. Keele (Eds.), Developing individuality in the buman brain: A tribute to Michael I. Posner (pp. 167-188). Washington, DC: American Psychological Association.

Rueda, M. R., Checa, P., \& Cómbita, L. M. (2012). Enhanced efficiency of the executive attention network after training in preschool children: immediate changes and effects after two months. Developmental cognitive neuroscience, 2, S192-S204. doi: 10.1016/j.den.2011.09.004

Rueda, F. J. M. (2013). Bateria Psicológica para Avaliação da Atenção (BPA). 1 a ed. São Paulo: Vetor.

Salen, K., \& Zimmerman, E. (2012). Regras do jogo: Fundamentos do design de jogos. São Paulo: Blucher.

Schmidt, M. E., \& Vandewater, E. A. (2008). Media and attention, cognition, and school achievement. The Future of children, 18(1), 63-85.

Sequeira, M. D. F. (1988). Os modelos de atenção e memória no processo de construção da leitura. Revista Portuguesa de Educação, 1(2), 73-79, 1988. Recuperado de http://repositorium.sdum.uminho.pt/ handle/1822/439

Shin, N., Sutherland, L. M., Norris, C. A., \& Soloway, E. (2012). Effects of game technology on elementary student learning in mathematics. British journal of educational technology, 43(4), 540-560. doi: 10.1111/j.1467-8535.2011.01197.x

Sigman, M., Peña, M., Goldin, A. P., \& Ribeiro, S. (2014). Neuroscience and education: Prime time to build the bridge. Nature neuroscience, 17(4), 497-502. doi: $10.1038 / \mathrm{nn} .3672$

Silva, L. N. O., Oliveira, M. F., \& Helene, A. F. (2014). Cognição e Esporte. Revista da Biologia. Recuperado de http://www.ib.usp.br/revista/node/159

Simonds, J., Kieras, J. E., Rueda, M. R., \& Rothbart, M. K. (2007). Effortful control, executive attention, and emotional regulation in 7-10-year-old children. Cognitive Development, 22(4), 474-488. doi: 10.1016/j.cogdev.2007.08.009

Stern, P., \& Shalev, L. (2013). The role of sustained attention and display medium in reading comprehension among adolescents with ADHD and without it. Research in developmental disabilities, 34(1), 431-439.

Strasburger, V. C., Wilson, B. J., \& Jordan, A. B. (2013). Children, adolescents, and the media. Pediatrics, 132 (5), 958-961.

Subrahmanyam, K., Greenfield, P., Kraut, R., \& Gross, E. (2001). The impact of computer use on children's and adolescents' development. Journal of Applied Developmental Psychology, 22(1), 7-30. 
Waters, A. M., Zimmer-Gembeck, M. J., Craske, M. G., Pine, D. S., Bradley, B. P., \& Mogg, K. (2015). Look for good and never give up: A novel attention training treatment for childhood anxiety disorders. Behaviour research and therapy, 73, 111-123.

Zhang, L., Yu, S., Li, B., \& Wang, J. (2017). Can Students Identify the Relevant Information to Solve a Problem? Journal of Educational Technology \& Society, 20(4), 288-299.

Zhao, J., Liu, M., Liu, H., \& Huang, C. (2018). The visual attention span deficit in Chinese children with reading fluency difficulty. Research in developmental disabilities, 73, 76-86.

Zheng, X., Swanson, H. L., \& Marcoulides, G. A. (2011). Working memory components as predictors of children's mathematical word problem solving. Journal of experimental child psychology, 110(4), 481-498.

Recebido em: 07/05/2018

Reformulado em: 02/10/2018

Aprovado em: 07/12/2018

Sobre a autora:

Daniela Karine Ramos é doutora em Educação, professora no Departamento de Metodologia de Ensino e no Programa de Pós-Graduação em Educação da Universidade Federal de Santa Catarina e líder do Grupo de Pesquisa Edumídia.

ORCID: http://orcid.org/0000-0001-9833-310X

E-mail:dadaniela@gmail.com

Contato com a autora:

Daniela Karine Ramos

Departamento de Metodologia de Ensino (MEN/CED) - Campus Universitário

Caixa Postal: 476 - Trindade

1 andar - Bloco B/CED

Florianópolis-SC, Brasil

CEP: 88040-900 